\title{
Distortion of Space and Time during Saccadic Eye Movements
}

\author{
Masataka Suzuki ${ }^{1}$, Yoshihiko Yamazaki ${ }^{2}$ \\ ${ }^{1}$ Department of Psychology, Kinjo Gakuin University, Nagoya, Japan \\ ${ }^{2}$ Department of Computer Science and Engineering Graduate School of Engineering \\ Nagoya Institute of Technology, Nagoya, Japan \\ Email:msuzuki@kinjo-u.ac.jp
}

\begin{abstract}
The space-time distortion perceived subjectively during saccadic eye movements is an associative phenomenon of a transient shift of observer's visual frame of reference from one position to another. Here we report that the lines of subjective simultaneity defined as two spatially separated flashes perceived during saccades were nearly uniformly tilted along the physical time-course. The causality of the resulting space-time compression may be explained by the Minkowski space-time diagram in physics.
\end{abstract}

Keywords: Saccade Space Time Compression

\section{Introduction}

In vision, an observer's frame of reference is one of the necessary elements of perceiving an event in space (where) and time (when). During saccadic eye movements, a space once recognized in one frame of reference is distorted toward the target as the eye fixates it in the new frame of reference [1,2]. Morrone et al. [3] demonstrated that the resulting space compression accompanies an underestimation of the perceived time interval between temporally separated stimuli. Since these two illusory effects occurred in nearly the same time range, they suggested the possibility of a single unifying mechanism of both the space and time compressions. Moreover, they speculated that the space-time compression is originated from anticipatory repositioning of visual receptive fields [4-6], leading to an immediate relativistic consequence in perceptual space and time [7]. To approach this mechanism, however, it is necessary to probe into the transient dynamics of these illusions [8]. In this study, we provide psychophysical evidence that the space-time compression during saccadic eye movements could be attributable to the backward temporal shift of time-course by which the observer perceives the saccade target in a new frame of reference. These effects on visual percepts of space, time and simultaneity may be explained along the framework of a 'thought experiment' of special relativity theory [9].

\section{Methods}

In this study, observers made a judgment as to the simultaneity of two briefly flashed stimuli appearing at different times during the course of a horizontal saccade (Figure 1(a)). The observers were seated in the dark, with their head fixed by a chin- and forehead-rest. Two-colored LEDs positioned on the black board in front of the observers (viewing distance: $40 \mathrm{~cm}$ ), were used as a central fixation target (FT) and a saccade target (ST) (panel 1 in Figure 1a). After fixating to the fixation target (FT) for a period of time between 1500 and $2500 \mathrm{~ms}$, both the FT and saccade targets (ST) were turned off simultaneously (pane 2 in Figure 1(a)), and the observers made a 30 degrees $\left({ }^{\circ}\right)$ horizontal saccade to the remembered ST as soon as both targets disappeared. Either of the FT or ST was also used to provide a standard stimulus (SS) or a comparison stimulus (CS), respectively. The early rising phase of electro-oculographic (EOG) signal less than $15 \%$ relative to its maximal value was used as a common triggering source (CTS) of SS and CS, each of which provides a flash with short exposure time $(1 \mathrm{~ms})$ at different latencies from CTS (e.g., see panel 3 in Figure 1(a)). The short exposure $(1 \mathrm{~ms})$ of flashed stimuli effectively minimized motion blur during saccades, allowing for the 
observers to specify the apparent positions of the two flashes.

In the first condition (Cond. 1), FT and ST provide SS and CS, respectively (e.g., see panel 3 in Figure 1(a)), while in the second condition (Cond. 2) they provide CS and SS, respectively. The SS was flashed at different constant latencies of $20,33,50$ or $100 \mathrm{~ms}$ from a CTS signal. In each latency condition, the experimenter initially set the time interval of CS and SS far below or far above the observer's threshold, and the observers were asked to adjust the variable timing of the CS on a trial-by-trial basis until it appeared equal to the latency of the SS, and to report corresponding spatial positions of the two flashes (e.g., see panel 4 in Figure 1(a)). In each of these ascending or descending sessions, the timing of the CS relative to that of SS was varied by the observer continuously via a dial on a pulse generator, and apparent position of two flashes perceived as simultaneous during the course of the saccade was pointed by the observer on a trial-by-trial basis, by adjusting the sensor head of a linear potentiometer (Novotechnik, TLH1000, length 0.8 $\mathrm{m})$, fixed sideways along a black board. In Cond.1 and 2, the subjective simultaneity of two flashes was estimated from the mean of five sets of ascending and descending sessions in each latency condition.

Horizontal eye position was recorded using an EOG system (AVH-10, Nihon-Koden) by placing $\mathrm{Ag}-\mathrm{AgCl}$ skin electrodes at the outer canthi of both eyes. A ground electrode was placed just above the eyebrows in the center of the forehead. As described earlier, the early rising phase of the EOG signal was used to trigger two pulse generators for the LED flashes of SS and CS. The base line adjustment of EOG signal was carried out carefully on a trial-by-trial basis. Target presentation and data collection were controlled using custom software programmed in LabVIEW (National Instruments). The eye position signals were digitally low-passed filtered at 50 $\mathrm{Hz}$, using a second-order Butterworth filter implemented in MatLab (The Mathworks). The onsets of eye movement were scored on the basis of $5 \%$ of the peak velocity of their position signals. Seven and six observers were used in Cond. 1 and Cond. 2, respectively.

\section{Results}

The spatial relationship between the perceived positions of the SS flashes and the corresponding eye positions was out-of-phase in both conditions (Figure 1(b), (c)). In Cond. 1, just after the instant of the saccade the SS appearing on FT was greatly mislocalized once nearest to the ST, but appeared near to the initial FT position as the eye fixated on the ST. In Cond. 2, the SS triggered $20 \mathrm{~ms}$ after the onset of CTS was invisible on ST, but in other

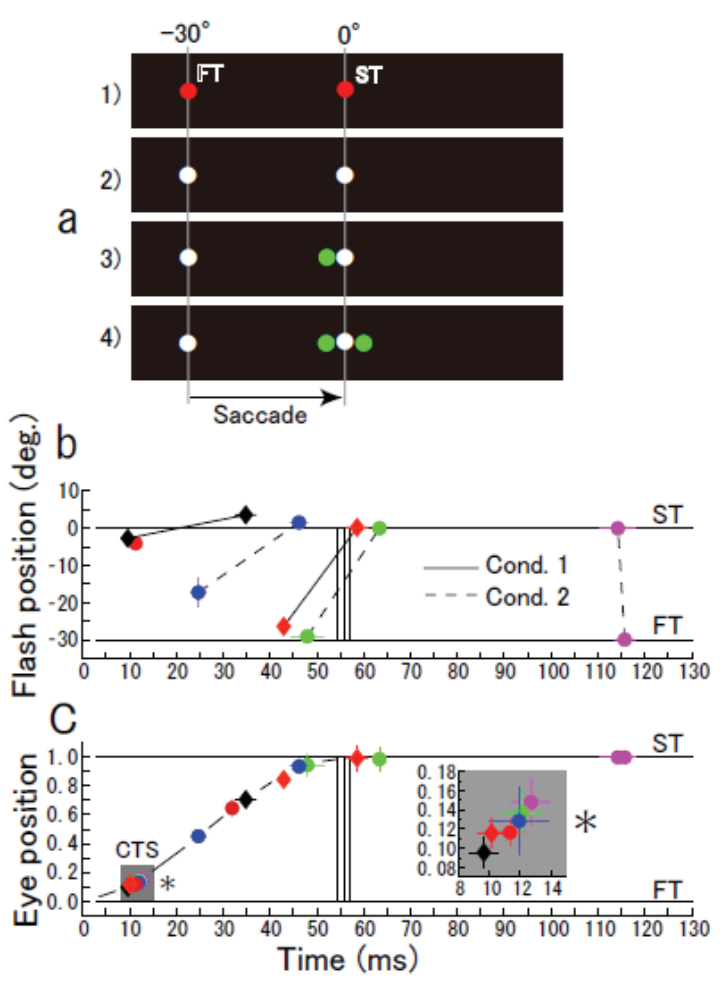

Figure 1. Subjective simultaneity of two flashes. (a) Spatial layout of two targets (FT, ST) and perceived flash stimuli specified on a black board. The same two-colored LEDs were used for both FT and ST targets. 1) FT (red) and ST (red) presented simultaneously at $-\mathbf{- 3 0}^{\circ}$ right and $0^{\circ}$ (screen center), respectively. 2) latency period from the simultaneous disappearance of two targets. White circles are not real, but are to refer the spatial position of the two targets. 3) a typical example of Cond. 1, showing that the spatial position of a FT flash (green) is greatly mislocalized toward the ST, and the ST flash triggered at the same time with the FT flash is invisible. 4) spatial positions of ST and FT flashes perceived as simultaneous. (b) Space-time diagram of two flashes perceived as simultaneous. The lines of subjective simultaneity in all latency conditions are represented by the solid and dashed lines in Cond.1 and Cond. 2, respectively. In Cond. 1, a pair of black or red diamonds represents the averaged estimate for the tasks when the latencies of SS flashes from the CTS signals are set at 0 or $33 \mathrm{~ms}$, respectively. In Cond. 2, similarly, red, blue, green and magenta circles represent the averaged estimate for the tasks when latencies of SS flashes from the CTS signals were set at 20 , 33,50 and $100 \mathrm{~ms}$, respectively. Note when SS latency from the CTS signal was set at $20 \mathrm{~ms}$, the flash on the ST was invisible and thus the CTS signal (red) is depicted alone. (c) The corresponding amplitudes of the EOG signal to the flash times of a pair of SS and CS in panel b. Each value is normalized to the magnitude at $100 \mathrm{~ms}$ after the movement ends. Small panel on the right (asterisk) shows enlarged representation of latencies and amplitudes of the CTS for all tasks. Note that the variations of CTS measures are limited in time and amplitudes across all tasks. Three vertical bars in panels b) and c) represent mean \pm s.d. of eye movement times for all tasks and subjects. 
tasks it was clearly identified, having a negative relationship to eye position, similar to the Cond.1.

In both conditions, when two stimuli were presented simultaneously, the ST flashes were perceived to occur earlier than the FT flashes across the saccadic period. Therefore, the observers estimated the simultaneity of the two flashes by delaying the onset time of the ST flash relative to that of FT in Cond. 1 (e.g., see panel 4 in Fig 1(a)), or by preceding the onset time of FT flash relative to the ST in Cond. 2 (Figure 1(b)). In Cond. 1, the time intervals of two flashes as an estimate of the subjectivesimultaneity averaged $25( \pm 2)$ and $16( \pm 1) \mathrm{ms}$, for SS set at 0 and $33 \mathrm{~ms}$ from the onset of CTS, respectively (solid lines). In Cond. 2 the ST at less than $30 \mathrm{~ms}$ after saccade onsets was invisible, so the same measures of subjective simultaneity of the two flashes were limited to the other three cases, averaging $22( \pm 1), 16( \pm 4)$ and 2 $( \pm 1) \mathrm{ms}$, for SS set at 33, 50 and $100 \mathrm{~ms}$ from the onset of CTS, respectively (dashed lines). In both conditions, therefore, the subjective simultaneity of two flashes can be referred to as the rightward tilt of the lines of simultaneity and as their directional uniformity across the saccadic period.

The effect of target eccentricity on perceiving simultaneity of two flashes was examined under static conditions without a saccade. The observers gazed $-30^{\circ},-15^{\circ}$ or $0^{\circ}$ relative to the $\mathrm{ST}$, and flash stimuli $(\mathrm{SS} / \mathrm{CS})$ were provided by two targets positioned at $-5^{\circ} / 5^{\circ},-15^{\circ} / 5^{\circ}$ or $-30^{\circ} / 0^{\circ}$, respectively. These spatial relationships between gaze directions and two flashes were roughly analogous to those in Cond. 2 (Figure 1 (b), (c)). Both stimuli were given by the experimenter, while the observers were asked to synchronize them by adjusting their interval on a trial-by-trial basis. Two flashes were apparently perceived in all tasks, and their intervals perceived as simultaneous averaged $2( \pm 2), 1( \pm 3)$ and $2( \pm 5) \mathrm{ms}$ at $-30^{\circ},-15^{\circ}$ and $0^{\circ}$ conditions, respectively. In the static condition, therefore, the effect of target eccentricity on estimating the subjective simultaneity of two flashes could be minor.

\section{Discussions}

In this study, we found that the lines of subjective simultaneity defined as two spatially separated flashes during saccades were nearly uniformly distorted on the physical time-course (Figure 1(b)). When interpreting this in perceptual space-time, however, two simultaneous events (flashes) must be on a line parallel to the space axis. This corresponds to the backward temporal shift of the time-course of ST flashes, relative to the FT flashes (Figure 2). When this shift component, herein termed $\Delta t$,

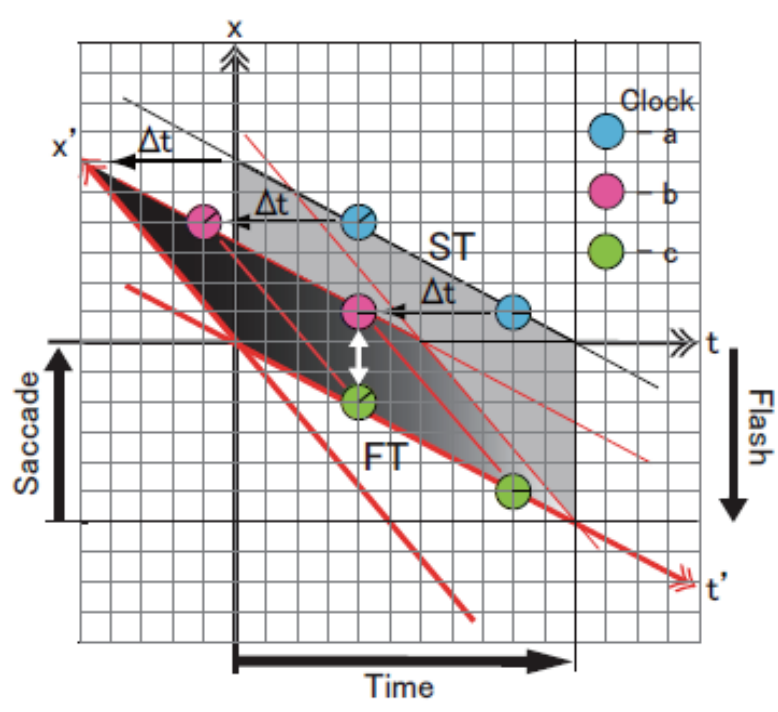

Figure 2. Schematic illustration of the space-time compression based on our results. Two coordinates, $\left(x, t^{\prime}\right)$ and $\left(x^{\prime}, t^{\prime}\right)$, are superimposed, analogues to the Minkowski space-time diagram. The first is Newtonian space-time coordinates to define position $(x)$ and time ( $\left.t^{\prime}\right)$ for a moving object(s) in real (physical) space-time, and the second is perceptual space-time coordinates $\left(x, t^{\prime}\right)$ hypothesized based on the present experiments. For details, see text.

is applicable over a saccadic period, the invisibility of the ST flashed during the initial half of the saccadic period (Figure 1(b)) could be ascribed to the backward temporal shift of the flash's percept beyond the extent of the conscious time window [10] by the amount $\Delta t$. Similarly, an earlier recovery time of the flash position to the ST than to the FT near the movement end may be explained by the same scheme. As for the latter, it is well established [11] that the target percept at the end of the saccade is referred backward in time to compensate for the time lost during saccadic suppression [12,13]. Of more importance in Figure 2 is the strong dependence of both the space and time compressions on the backward shift of the time-course of the ST flashes. This scheme is different from the convergent type of compression proposed previously [1-3].

Among these, Morrone et al. [3] have shown that time compression perceived subjectively during saccadic eye movements is an associative phenomenon of space compression, which was evidenced as a convergent type of mislocalization of visual stimuli toward the endpoint of the saccade. They suggested that the resulting alternation of spatial and temporal metrics of perceptual space-time would lead to relativistic-like effects on the visual percept [7]. Our results, by contrast, indicate that space and time compressions could be attributable to the shift component of mislocalization of visual stimuli. To illustrate this, as shown in Figure 2, we define Newtonian 
space-time coordinates $\left(x, t^{\prime}\right)$, where the tilted time axis $t$ ' is to specify the corresponding position $(x)$ of two flashes, both moving in the opposite direction to the saccadic eye movement. Since in Newtonian space-time the geometry of space is Euclidian and the time is universal for all observers, the simultaneity of two flashes is specified on the line parallel to the space axis $x$ (e.g., see clock $a$ and $c$ ). However, this was not the case in the perceptual space-time of our observers. According to the rightward tilt of the lines of simultaneity over a saccadic period in figure $1 b$, the sequence of events from the viewpoint of our observer may be illustrated graphically by shifting the timescale on the ST in the diagram backward by an amount $\Delta t$. This corresponds to a tilt in the space axis from vertical $(x)$ to leftward ( $x$ ': red lines). Note this lead us to define another space-time coordinates $\left(x^{\prime}, t^{\prime}\right)$, in which a hypothetical observer moving with this frame of reference, sees all events occurring on a line parallel to the space axis $x$ ' as simultaneous (e.g., see clock $b$ and $c$ ). The superimposed representation of two coordinates, $(x$, $\left.t^{\prime}\right)$ and $\left(x^{\prime}, t^{\prime}\right)$, and their interrelationship are analogues to the Minkowski space-time diagram in special relativity theory [9]. Thus, considering our results from the viewpoint of our observer, three relativistic-like effects, the lack of absolute simultaneity, space contraction (compression) and time compression, can be expected. First, the fact that the observers estimated the simultaneity of two flashes by delaying the time of the ST flash relative to that of FT by the amount $\Delta t$ suggests that during saccadic periods the observers see the two flashes occurring in the space-time coordinates $\left(x^{\prime}, t^{\prime}\right)$. As a result, the two flashes perceived as simultaneous in $\left(x^{\prime}, t^{\prime}\right)$ are not simultaneous in $\left(x, t^{\prime}\right)$. Second, if this was the case, as shown by the distance of two white arrow heads in the figure, the spatial distances of the two flashes appear to contract in the direction of motion (space compression). Third, for this observer, all events happening on the moving ST flashes are compressed relative to that on the FT (time compression), or the time passed on the clock $c$ is dilated relative to time passed on the clock $b$, by the amount $\Delta t$. Taken together, what is novel here is to present a single unifying mechanism of space and time compression using the Minkowski diagram [9], in which the space-time compression during saccades could be ascribed to the homogeneous distortion of space along a time scale, rather than the convergent type of compression proposed by Morrone et al. [3].

\section{Conclusions}

In the present study, the pattern of space-time distortion perceived subjectively during saccadic eye movements was studied in order to gain insights into the nature of corresponding space-time compression inherent in human visual perception. We found that the lines of subjective simultaneity defined as two spatially separated flashes perceived during saccades were nearly uniformly tilted along the physical time-course. This tempted us to speculate that vision may be subject to relativistic effects, similar to physical relativistic effects that occur at speeds approaching the speed of light. It is well established that neuron's receptive fields or their representation of space are not static entities but that they start to change perisaccadically to bring a visual stimulus defined in presaccadic frame of reference into a post-saccadic frame of reference [4-6]. When this dynamic coordinate transformation is rapid, approaching the physical limit of neural information transfer, the relativistic consequences may be expected.

\section{References}

[1] M. Lappe, H. Awater and B. Krekelberg, "Postsaccadic visual references generate presaccadic compression of space," Nature 403, pp. 892-895, 2000.

[2] J. Ross, M. C. Morrone, and D. C. Burr, "Compression of visual space before saccades," Nature 384, pp. 598-601, 1997.

[3] M. C. Morrone, J. Ross, and D. C. Burr, "Saccadic eye movements cause compression of time as well as space," Nature Neuroscience 8, pp. 950-954, 2005.

[4] J. R. Duhamel, C. L. Colby, and M. E. Goldberg, "The updating of the representation of visual space in parietal cortex by intended eye movements," Science 255, pp. 90-92, 1992.

[5] M. Kusunoki and M. E. Goldberg, "The time course of perisaccadic receptive field shifts in the lateral intraparietal area of the monkey," Journal of Neurophysiology 89, pp. 1519-1527, 2003.

[6] M. M. Umeno and M. E. Goldberg, "Spatial processing in the monkey frontal eye field. I. Predictive visual responses," Journal of Neurophysiology 78, pp. 1373-1383, 1997.

[7] M. C. Morrone, J. Ross, and D. C. Burr, "Keeping vision stable: rapid updating of spatiotopic receptive fields may cause relativistic-like effects," In R. Nijhawan (Ed.), Space and time in perception and action, Cambridge: Cambridge University Press, 2008.

[8] D. M. Eagleman, "Distortion of time during rapid eye movements," Nature Neuroscience 8, pp. 850-851, 2003.

[9] A. Einstein, "Relativity: The Special and General Theory," New York: Henry Holt, 1920.

[10] B. Libet, E. W. J. Wright, B. Feinstein, and D. K. Pearl, "Subjective referral of the timing for a conscious sensory experience: a functional role for the somatosensory specific projection system in man,” Brain 102, pp. 193-224, 1979.

[11] K. Yarrow, P. Haggard, R. Heal, P. Brown, and J. C. Roth- 
well, "Illusory perceptions of space and time preserve cross-saccadic perceptual continuity," Nature 414, pp. 302-305, 2001.

[12] M. R. Diamond, J. Ross, and M. C. Morrone, "Extraretinal control of saccadic suppression," Journal of Neuro- science 20, pp. 3442-3448, 2000.

[13] M. C. Morrone, J. Ross, and D. C. Burr, "Apparent position of visual targets during real and simulated saccadic eye movements," Journal of Neuroscience 17, pp. 79417953, 1997. 